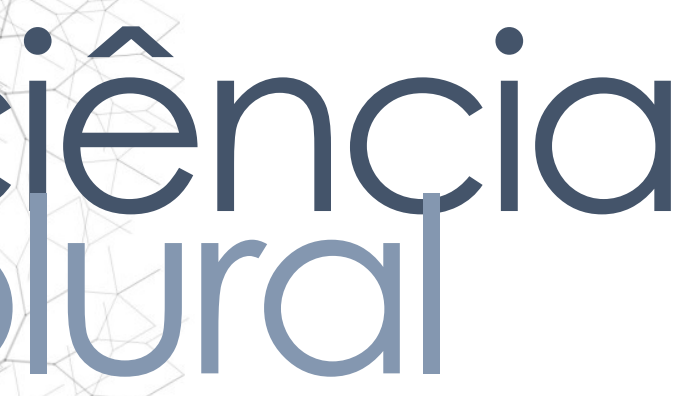

\title{
PROTOCOLO CLÍNICO E LABORATORIAL NA REABILITAÇÃO ORAL COM PRÓTESE OBTURADORA E SATISFAÇÃO DO PACIENTE: CASO CLÍNICO
}

\author{
Clinical and laboratory protocol in oral rehabilitation with shutter \\ prosthesis and patient satisfaction: clinical case
}

Protocolo clinico y de laboratorio en rehabilitación oral con prótesis de obturación y satisfacción del paciente: caso clínico

Laércio Almeida de Melo • Universidade Federal do Rio Grande do Norte • Doutor em Saúde Coletiva • Professor Adjunto no Departamento de Odontologia da UFRN - E-mail: laercio_melo91@hotmail.com

Marcos Paulo Miranda Silva • Universidade Federal do Rio Grande do Norte • Cirurgião-dentista • E-mail: marcosatendimento@hotmail.com

José Henrique de Souza Moura • Universidade Federal do Rio Grande do Norte • Cirurgião-dentista • E-mail: henrique-cp@hotmail.com

Erika Oliveira de Almeida • Universidade Federal do Rio Grande do Norte • Doutora em Prótese Dentária • Professora Adjunto no Departamento de Odontologia da UFRN • E-mail: erika.almeida.protese@gmail.com

Adriana da Fonte Porto Carreiro • Universidade Federal do Rio Grande do Norte • Doutora em Prótese Dentária • Professora Associado no Departamento de Odontologia da UFRN • E-mail: adrianadafonte@hotmail.com

Autor responsável pela correspondência:

Laércio Almeida de Melo • E-mail: laercio_melo91@hotmail.com 


\section{RESUMO}

Introdução: A enorme dificuldade de deglutição e fala apresentada em pacientes que sofreram maxilectomia total ou parcial por consequência de patologias bucais, afeta grande parte da população que realizou tal procedimento. A alternativa de minimizar o impacto causado pela sequela deixada após cirurgia em maxila está na confecção de um obturador palatino, que tem como objetivo obstruir a comunicação oronasal. Objetivo: Este trabalho objetivou descrever um relato de caso evidenciando o passo a passo clínico e laboratorial de uma prótese total obturadora e a satisfação do paciente com a mesma. Método: Paciente do gênero masculino, maxilectomizado há seis anos devido a presença de um Carcinoma Adenóide Cístico foi reabilitado com prótese total obturadora superior e prótese total convencional inferior. A reabilitação superior seguiu os mesmos passos de uma prótese total convencional, com exceção da moldagem funcional, na qual foi feita a moldagem da comunicação bucosinusal com silicona de condensação antes da moldagem do selado periférico e moldagem funcional propriamente dita. Na etapa laboratorial, foi necessária a realização de três prensagens a fim de deixar a prótese oca na porção obturadora. Resultados: $\mathrm{O}$ tratamento restabeleceu a dimensão vertical perdida, proporcionou uma oclusão adequada, se mostrou melhor esteticamente e o paciente mostrou-se extremamente satisfeito com a reabilitação. Conclusões: Como conclusão, obteve-se uma prótese total obturadora superior com qualidades funcionais e estéticas satisfatórias. Ademais, ao comparar com a antiga, a nova mostrou-se satisfatória de acordo com a percepção do usuário e possui um menor peso, favorecendo uma melhor retenção.

Palavras-Chave: Obturador palatino; Cirugia bucal; Reabilitação.

\section{ABSTRACT}

Introduction: The enormous difficulty in swallowing and speaking presented in patients who underwent total or partial maxillectomy as a result of oral pathologies affects a large part of the population that performed this procedure. The alternative of minimizing the impact caused by the sequelae left after maxillary surgery is by making a palatal obturator, which aims to obstruct oronasal communication. Objective: This study aimed to describe a case report highlighting the clinical and laboratory step by step of a complete obturator prosthesis and patient satisfaction with it. Methods: A male patient who had been maxillectomized for 6 years due to the presence of a cystic adenoid carcinoma was rehabilitated with superior obturator total denture and inferior conventional total denture. The superior rehabilitation followed the same steps of a conventional total prosthesis, except for the functional molding, in which e bucosinusal communication with condensation silicon was molded before the eripheral sealing molding and the functional molding itself. In the laboratory stage, was necessary to perform three presses in order to leave the hollow prosthesis in the turator portion. Results: The treatment restored the lost vertical dimension, vided an adequate occlusion, was better aesthetically and the patient was tremely satisfied with the rehabilitation. Conclusions: In conclusion, a superior total 
obturator prosthesis with satisfactory functional and aesthetic qualities was obtained. Moreover, when compared to the old one, the new one was satisfactory according to the user's perception and has a lower weight, favoring a better retention.

Keywords: Palatal shutter; Oral surgery; Rehabilitation.

\section{RESUMEN}

Introduccion: La enorme dificultad para tragar y hablar presentada en pacientes que han sufrido una maxillectomía total o parcial como resultado de patologías orales afecta a gran parte de la población que realizó este procedimiento. La alternativa de minimizar el impacto causado por las secuelas que quedan después de la cirugía maxilar es hacer un obturador palatino, que tiene como objetivo obstruir la comunicación oronasal. Objetivo: Este estudio tuvo como objetivo describir un informe de caso que muestra la clínica y el laboratorio paso a paso de una prótesis obturadora completa y la satisfacción del paciente con ella. Método: Un paciente masculino que había sido maxillectomizado durante seis años debido a la presencia de un carcinoma adenoide quístico fue rehabilitado con una prótesis obturadora total superior y una prótesis total inferior convencional. La rehabilitación superior siguió los mismos pasos de una prótesis total convencional, excepto el moldeado funcional, en el cual la comunicación bucosinusal con silicio de condensación se moldeó antes del moldeado de sellado periférico y el moldeado funcional en sí. En la etapa de laboratorio, fue necesario realizar tres prensas para dejar la prótesis hueca en la porción del obturador. Resultados: El tratamiento restableció la dimensión vertical perdida, proporcionó una oclusión adecuada, fue mejor estéticamente y el paciente quedó extremadamente satisfecho con la rehabilitación. Conclusiones: Como conclusión, se obtuvo una prótesis obturadora superior con cualidades funcionales y estéticas satisfactorias. Además, en comparación con el anterior, el nuevo fue satisfactorio de acuerdo con la percepción del usuario y tiene un peso menor, lo que favorece una mejor retención.

Palabras clave: obturador palatino; Cirugía oral; Rehabilitación 


\section{Introdução}

A reabilitação oral com prótese obturadora é um método bastante adequado na reabilitação de pacientes que realizaram ressecção cirúrgica na maxila ou mandíbula devido à presença de tumores odontogênicos. Tradicionalmente, a obturação protética tem sido a modalidade de tratamento padrão para reabilitação palatina permitindo o fechamento imediato da comunicação oronasal. A prótese obturadora é de fácil fabricação e custo-benefício, minimizando o impacto emocional, estresse psicossocial sobre o paciente, alcançando resultados funcionais e estéticos satisfatórios ${ }^{1,2}$.

Em virtude da necessidade de reabilitação em pacientes que foram maxilectomizados, é importante a verificação das técnicas para confecção de próteses obturadoras. É imprescindível verificar o grau de extensão do obturador na parte defeituosa, o caráter do tecido de revestimento e os requisitos funcionais para retenção, suporte e estabilidade da prótese. Em defeitos grandes, o obturador é estendido verticalmente em grandes dimensões para selar o defeito cirúrgico e horizontalmente para nivelar os tecidos rebaixados, afetando seu tamanho e peso. Dessa forma, deve-se ficar atento ao peso do obturador, uma vez que em grandes proporções a prótese pode tornar-se desconfortável e não retentiva para o paciente, comprometendo sua função ${ }^{3,4}$.

Quando bem elaborada, uma prótese obturadora objetiva restaurar as funções da mastigação, deglutição e fala, proporcionando uma satisfação e melhora na qualidade de vida dos usuários. Outro benefício da reabilitação protética da maxilectomia a partir da reconstrução de tecidos autógenos, é que esse tratamento simplifica os exames oncológicos para possíveis identificação da recorrência do tumor ${ }^{5-8}$.

Mediante os resultados positivos que uma reabilitação facial, funcional e stética do paciente maxilectomizado pode trazer, um relato de caso de etapas clínicas laboratoriais é extremamente relevante para facilitar a confecção de próteses turadoras por cirurgiões-dentistas. Tal tratamento é caracterizado por ser raro e ssuir um passo a passo mais sensível em relação a outras próteses, o que indica a essidade de protocolos detalhados para a sua realização. 


\section{ciência
purâl}

Neste sentido, o presente trabalho tem como objetivo descrever um relato de caso destacando as etapas clínicas e laboratoriais no tratamento reabilitador com prótese obturadora e avaliar o impacto da reabilitação na autopercepção da satisfação com o tratamento.

\section{Metodologia}

\section{Apresentação do caso}

Paciente S.R.F., do sexo masculino, 60 anos, compareceu ao departamento de Odontologia da Universidade Federal do Rio Grande do Norte com queixa de próteses folgadas e mal adaptadas. O mesmo foi maxilectomizado há seis anos devido a um Carcinoma Adenóide Cístico. Tal cirurgia lhe ocasionou a perda de estruturas da hemiface esquerda, caracterizada por retirada de partes ósseas e moles da cavidade nasal, do seio maxilar, órbita e porção esquerda do osso maxilar, palato e cornetos nasais. No exame clínico, percebeu-se que o mesmo fazia uso de uma prótese total obturadora palatina pesando $30,7 \mathrm{~g}$ e de uma prótese total convencional inferior, ambas com cinco anos de uso. As próteses apresentavam-se com higiene pobre, sem retenção, desgastadas e sem planos oclusais bem definidos (Figura 1). O paciente também apresentava perda de Dimensão Vertical de Oclusão (DVO).

Figura 1. Próteses antigas em oclusão.

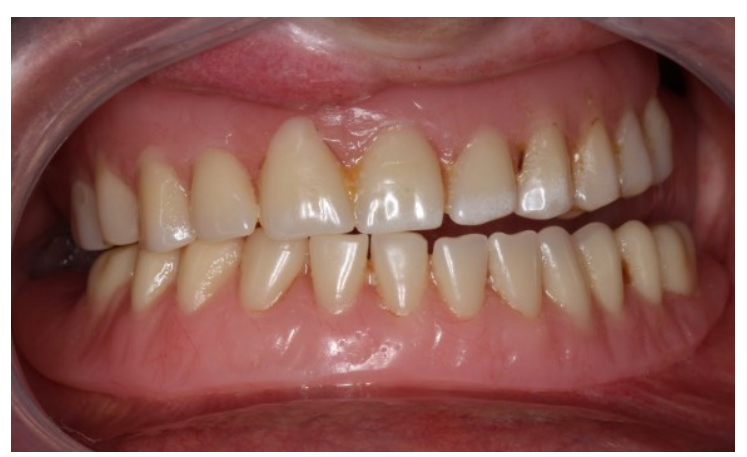

Fonte: Autores, 2019. 


\section{Plano de tratamento}

Após uma avaliação do caso e do consentimento do paciente, foi dado início a confecção da prótese total obturadora superior e convencional inferior. Os passos clínicos para confecção da prótese obturadora não foi diferente daqueles para fazer uma prótese total convencional (exame clíncico, moldagem anatômica, moldagem funcional, ajuste do plano de orientação, registro das relações maxilomandibulares, prova dos dentes em cera e instalação). No entanto, alguns cuidados específicos (utilização de materiais menos fluidos e realização de três prensagens) na moldagem funcional da cavidade presente e etapas laboratoriais para tornar a parte da prótese obturadora oca foram tomadas.

\section{Autopercepção da satisfação}

A autopercepção de satisfação com relação às próteses antigas e novas foi aferida a partir de uma questão: "Como você avalia seus dentes, gengivas ou próteses?”, com cinco respostas possíveis, “Péssimas”, “Ruins”, “Regulares”, “Boas” e "Excelentes". Tal pergunta é capaz de predizer, individualmente, o Geriatric Oral Health Assessment Index (GOHAI), instrumento amplamente utilizado para avaliar a autopercepção de saúde bucal ${ }^{5}$. Antes do início do tratamento e ao final, foi perguntado ao paciente o grau de satisfação da sua condição bucal.

\section{Avaliação da retenção}

Avaliou-se a retenção antes da reabilitação com as próteses antigas e três meses após a instalação das próteses novas. Essa retenção foi avaliada através do método proposto por Limpuangthip et al. ${ }^{9}$. Para a maxila, uma retenção foi considerada adequada quando houve a necessidade de se aplicar uma força maior ou igual a 5N para o seu deslocamento vertical. Já para a mandíbula, a retenção foi dada como ideal quando a força para deslocar a prótese foi igual ou superior a 2,5N. Essa retenção é verificada colocando o polegar e o indicador na face vestibular e lingual dos cisivos centrais e, em seguida, uma força de tração vertical é feita para realizar o locamento. Para se ter ideia da força aplicada, o pesquisador calibrou sua força de ção em uma balança de precisão. 
Essa variável também foi estimada através de níveis, e a retenção foi considerada boa, quando se aplicou uma força maior que $10 \mathrm{~N}$ para o deslocamento; moderada, quando houve a necessidade de uma força entre 5 e 10N; mínima, com uma força entre 2,5 e $5 \mathrm{~N}$ e ausência de retenção quando o deslocamento se deu com o paciente em repouso.

\section{Resultados e Discussão}

Como resultado, o tratamento restabeleceu a dimensão vertical perdida, proporcionou uma oclusão adequada e se mostrou melhor esteticamente. Anteriormente ao tratamento, o paciente relatou que as condições das suas próteses estavam péssimas. Já com relação às próteses novas, quando questionado sobre sua autopercepção de satisfação com elas, relatou estar excelente. O protocolo seguindo todas as etapas clínicas adequadas para uma prótese total, sem pular nenhuma etapa, favoreceu uma maior retenção da nova prótese com relação à antiga, principalmente pela característica peculiar do bulbo ou tampão obturador ser oco. Diante da realização de uma prótese obturadora oca, foi possível diminuir o peso da prótese antiga $(30,7 \mathrm{~g})$ em 2,1g, obtendo uma nova com $28,6 \mathrm{~g}$.

No relato de caso descrito neste estudo, a prótese obturadora teve como propósito melhorar a estética e a função mastigatória, a partir de uma melhor retenção da peça protética. A prótese obturadora é o principal mecanismo para reabilitar defeitos morfofuncionais, visto que visa fechar o defeito maxilar, separando a cavidade bucal da cavidade nasal, evitando a fala hipernasal e regurgitação nasal por alimentos ou líquidos. Tais fatos proporcionam a melhora da função mastigatória, da estética, da aceitação psicológica, ressocialização e sustentação ao perfil facial 5 . Como consequência desses benefícios, esse tipo de reabilitação tem a capacidade de proporcionar uma superação do trauma sofrido pela cirurgia, impactando uma melhora na qualidade de vida. Além disso, esses indivíduos reabilitados, anteriormente com vergonha de si mesmos, acabam voltando ao convívio social com amigos e familiares devido a uma satisfação estética que a prótese obturadora é capaz de fornecer. 
A literatura relata que técnicas de confecção para prótese obturadora é diferente da prótese convencional, pois a sua área protética é bastante elevada. Dessa forma, após uma moldagem preliminar com um material hidrocolóide irreversível, recomenda-se que na moldagem funcional, realize-se a impressão da comunicação buco sinusal com silicone de condensação ou adição previamente ao selado periférico e moldagem funcional propriamente, utilizando uma moldeira individual ${ }^{6}$. No presente estudo, tal sequência foi adotada mediante a sequela da maxilectomia abranger quase a metade do palato. Além dessa diferença clínica, dentro das etapas laboratoriais, houve-se a necessidade da realização de três prensagens da prótese maxilar, com o intuito de se obter uma prótese oca na região obturadora.

\section{Etapas clínicas para a confecção da prótese obturadora}

No arco superior, realizou-se a moldagem anatômica com moldeira perfurada para desdentado total compatível com o tamanho da cavidade bucal do paciente (Figura 2A). O material utilizado para essa moldagem foi o alginato (Hydrogum 5 - Zhermack). Tal escolha baseou-se na boa fluidez desse material, o que proporciona boa adaptação aos tecidos bucais sem causar deslocamento dos mesmos.

Figura 2. Moldagem anatômica com alginato.

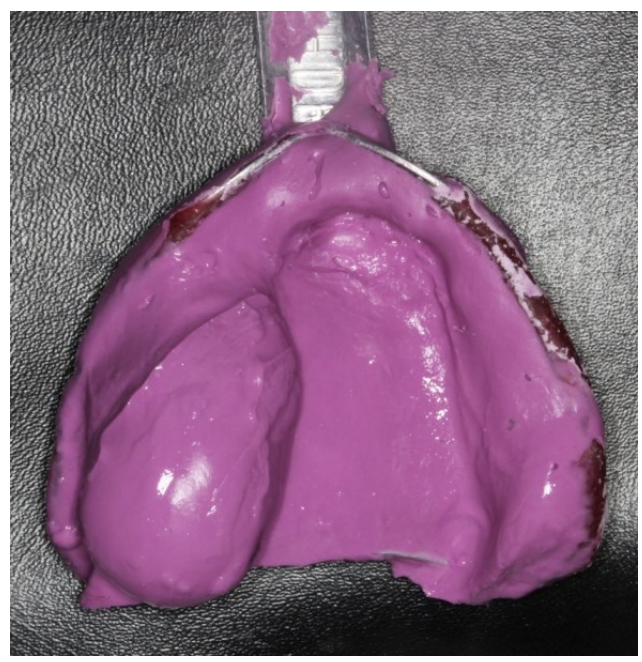

Fonte: Autores, 2019.

Para a moldagem funcional, inicialmente foi colocado na moldeira individual furada uma porção de silicona de condensação (Zetalabor - Zhermack) para 


\section{ciênncia
piural}

moldar a comunicação bucosinusal. Esse procedimento possibilitou uma reprodução fiel da comunicação bucosinusal previalmente a moldagem funcional. Em seguida, utilizou-se a godiva para a moldagem do selado periférico (Figura 3A). Essa moldagem permite uma maior extensão possível da futura prótese cobrindo toda a área chapeável. No intuito de finalizar esse tipo de moldagem para copiar de forma mais fiel toda a área chapeável e a abertura bucosinusal, foi utilizado um poliéter (Impregum - 3M) para realizar a moldagem funcional propriamente dita (Figura 3B).

Figura 3. Moldagens do selado periférico, da comunicação bucosinusal e funcional propriamente dita.

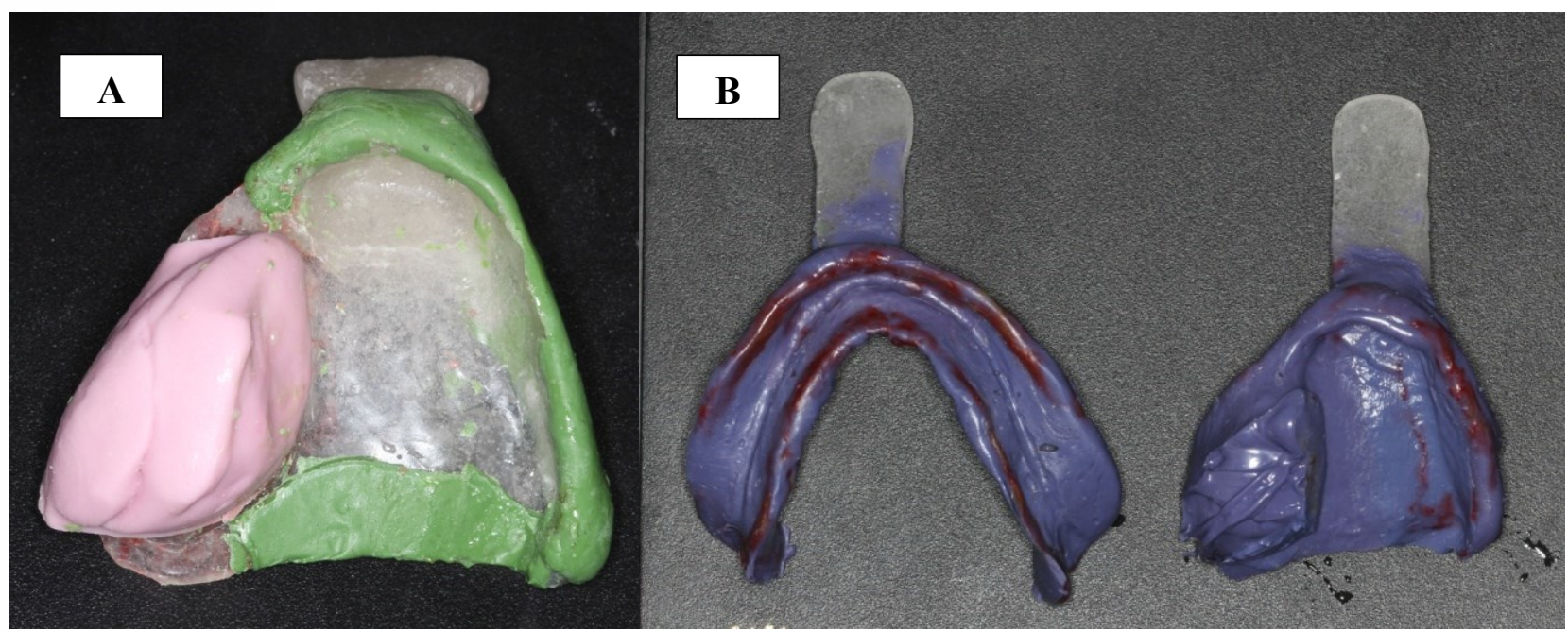

Fonte: Autores, 2019.

Legenda: A - Moldagens periférica e da comunicação bucosinusal. B - Moldagem funcional propriamente dita.

Esse material foi utilizado por ser de alta precisão, possuir um bom escoamento para moldar os tecidos moles e por ter uma alta estabilidade, o que diminui a ocorrência de alteração dimensional do material e consequentemente menores distorções da anatomia real do paciente.

A posteriori, foi realizado o ajuste do plano de orientação superior, seguindo os eguintes passos: ajuste do suporte de lábio, altura do plano oclusal anterior, altura do ano oclusal posterior, corredor bucal e linhas de referências. O ajuste da altura erior e posterior do plano oclusal foi realizado com o auxílio da régua de Fox, que composta de duas partes, sendo uma intraoral e outra extra oral. A parte extra oral régua de Fox é posta sobre os pontos referências extra orais para guiar o paralelismo 


\section{ciência plural}

a ser obtido com o plano oclusal. A parte intraoral deve ser colocada sobre a superfície oclusal do plano de orientação, observando-se através da sua porção extra oral o paralelismo com a linha bipupilar, na região anterior, e com o plano de Camper (plano correspondente a uma linha que une a borda inferior da asa do nariz à porção média do trágus), na região posterior.

Após essa etapa, foi realizado o ajuste do plano de orientação inferior. Sendo assim, a Dimensão Vertical de Oclusão através da associação dos métodos métrico, fonético e estético foi determinada ${ }^{10-12}$. A relação cêntrica também foi estabelecida a partir da associação dos métodos de manipulação e fisiológico, que posteriormente foi registrada para a montagem em articulador dos planos de orientação ${ }^{10}$. Para a realização do registro, o paciente foi colocado em relação cêntrica (RC), na qual a mandíbula do paciente é manipulada para uma posição mais posterior ao mesmo tempo em que se pede para ele colocar a ponta da língua no palato. Uma vez determinada à posição de $\mathrm{RC}$, os planos de orientação foram fixados com o auxílio de grampos de papéis aquecidos e encaixes no plano de cera superior utilizando para os seus preenchimentos o impregum (Figura 4). Logo em seguida, os modelos registrados foram montados no articulador com o auxílio da mesa de camper.

Figura 4. Registro das relações maxilomandibulares.

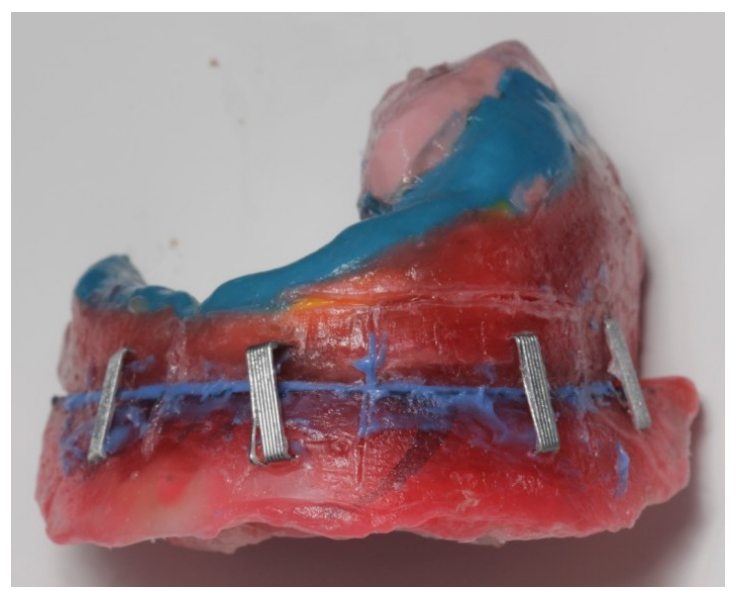

Fonte: Autores, 2019.

Posteriormente, ocorreu a prova dos dentes em cera. Nessa etapa, foi firmada todas as características estéticas (suporte do lábio, altura anterior e terior do plano oclusal, corredor bucal e linha média) e funcionais (oclusão) vvadas no ajuste do plano de orientação. Além disso, essa etapa foi de suma 
importância para o paciente visualizar conforme mostrado na figura 5 o formato e cor dos dentes artificiais da futura prótese (Figura 5-A). Por fim, após a acrilização, foi feita a instalação da prótese obturadora e o ajuste oclusal (Figuras 5-B).

Figura 5: Visualização do formato e cor dos dentes (5-A) e instalação da prótese obturadora com ajuste oclusal (5-B)

Figura 5-A.

Figura 5-B.

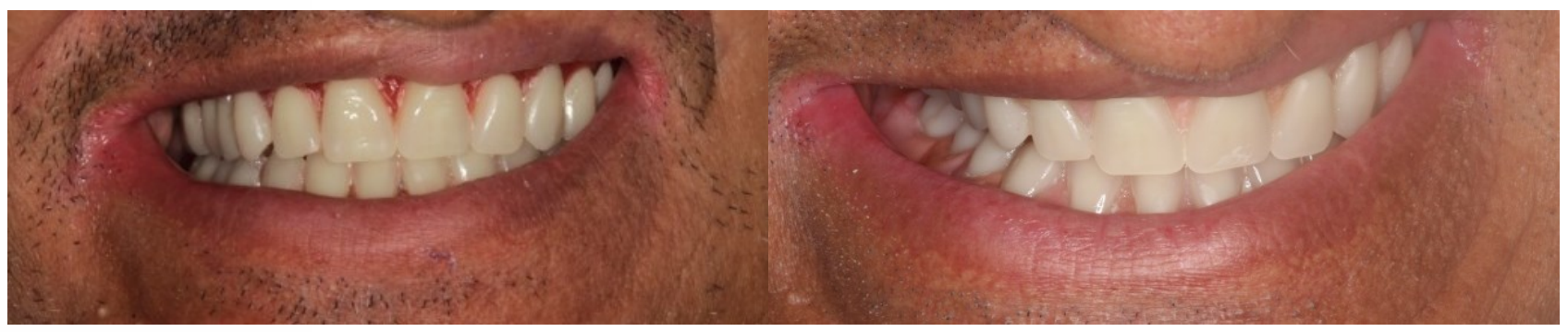

Fonte: Autores, 2019.

\section{Etapas laboratoriais para a confecção da prótese obturadora}

Com o modelo anatômico vazado, procedeu-se a realização da moldeira individual utilizando resina acrílica autopolimerizável transparente. Essa moldeira individual é de suma importância para o acondicionamento do material de moldagem que dará origem ao modelo funcional. Ela é confeccionada numa extensão de 3mm aquém do assoalho bucal, permitindo assim que a godiva tenha espaço para moldar o fundo de saco com maior precisão.

Em seguida, após a moldagem funcional feita pelo clínico, foi relaizado no laboratório a dicagem do molde com cera utilidade. É nesse procedimento que as futuras bordas da prótese foram preservadas e delimitadas. Feito isso, o vazamento do molde com gesso pedra foi realizado e em seguida o plano de orientação (base de prova e plano de cera) foi confeccionado. Assim como na moldeira individual, antes da realização dos planos de orientação, o modelo funcional foi aliviado com cera 7. No modelo anatômico para a confecção da moldeira individual, as rugas e suturas alatinas, mucosa flácida, áreas retentivas e a região interna da abertura buco-sinusal oram aliviados. Na realização do plano de orientação, as áreas retentivas, incluindo a rtura buco-sinual também foram aliviados. 
A confecção laboratorial da prótese obturadora segue alguns critérios que são de extrema importância para a obtenção de um tampão obturador eficiente. Durante a etapa de inclusão é importante a confecção de duas bases para obter o bulbo ou tampão oco, favorecendo um menor peso da prótese e consequentemente maior estabilidade da prótese. Dessa forma, é necessária a realização de três prensagens, uma com a base da prótese, outra para o tampão e uma última com o tampão e a base da prótese juntos. Para verificar o vedamento do obturador, o teste utilizado foi a imersão da prótese dentro d'água. Ao imergir a peça, não foi visto a entrada de água na porção oca, o que confirmou o vedamento da prótese. As etapas seguintes são as mesmas propostas para um a prótese total (polimerização, ajuste oclusal, acabamento e polimento).

\section{Conclusão}

Foi possível a obtenção de uma prótese obturadora maxilar leve, com boa retenção, estabilidade e estética. Além disso, ao comparar com a prótese antiga, a reabilitação proposta por este estudo mostrou-se satisfatória de acordo com a percepção do usuário. Tais achados foram possíveis graças a um seguimento rigoroso das etapas clínicas e laboratoriais necessárias para a confecção desse tipo de prótese.

\section{Referências}

1. Badadare MM, Patil SB, Bhat S, Tambe A. Comparison of obturator prosthesis fabricated using different techniques and its effect on the management of a hemipalatomaxillectomy patient. BMJ Case Rep. 2014; 21.

2. Rani S, Gupta S, Verma M. Hollow bulb one piece maxillary definitive obturator - A simplified approach. Contemp Clin Dent. 2017;8(1):167-170.

3. Ali MM, Khalifa N; Alhajj MN. Quality of life and problems associated with obturators of patients with maxillectomies. Head Face Med. 2018;14(1):1-9.4. Dummel Cardoso FB, Rivero LF, Martins MD, Zieger RA, Ribeiro JT, Martins AT. Ameloblastic carcinoma of the maxilla in a young woman: from diagnosis to ehabilitation. Oral Surg Oral Med Oral Pathol Oral Radiol. 2020;129(1):e21.

5. Dholam KP, Chouksey GC, Dugad J. Oral health-related quality of life after prosthetic rehabilitation in patients with oral cancer: A longitudinal study with the Liverpool Oral Rehabilitation Questionnaire version 3 and Oral Health Impact Profile14 questionnaire. Indian J Cancer. 2016;53(2):256-260. 
6. Hattori M, Sumita YI, Taniguchi H. Measurement of Voice Onset Time in Maxillectomy Patients. Sci World J. 2014;2014;1-4.

7. Chen C, Ren W, Gao L, Cheng Z, Zhang L, Li S, Zhi PK. Function of obturator prosthesis after maxillectomy and prosthetic obturator rehabilitation. Braz J Otorhinolaryngol. 2016;82(2):177-183.

8. Aguiar L, Mozzini AR, Lersch E, Conto F. Obturador palatino: confecção de uma prótese não convencional - relato de caso. RFO UPF. 2013;18(1):125-129.

9. Limpuangthip N, Somkotra T, Arksornnukit M. Modified retention and stability criteria for complete denture wearers: A risk assessment tool for impaired masticatory ability and oral health-related quality of life. J Prosthet Dent. 2018;120(1):43-49.

10. Telles D. Prótese Total: Convencional e Sobre Implantes. Santos: Santos, 2009. 492 p.

11. Varghese K. Prosthetic Rehabilitation of a Congenital Soft Palate Defect. J Indian Prosthodont Soc. 2012;14(1):181-186.

12. Miyashita ER. Sistemas de Retenção em Prótese Obturadora Cirúrgica Para Pacientes Maxilectomizados. Pesquisa Brasileira em Odontopediatria e Clínica Integrada. 2011;11(2):263-268. 